\title{
Research on the General Situation of College Students' Coping Styles
}

\section{Zhihui Gong}

Jilin Engineering Normal University, Changchun City, Jilin Province, China

This article comes from a school-level scientific research project: In the era of "Internet +", the innovation research on the training model of secondary vocational education in Jilin Province. The project number is: XYB201841.

This paper is the stage research result of 2020 Doctoral project of Jilin Engineering Normal University(BSGC202007)

Abstract: Coping styles are the adjustment behavior of college students to stressful situations. To explore the General Situation of college students' coping styles, use the simple coping style questionnaire compiled by Xie, Yaning in 1998 to conduct a survey. The questionnaire is divided into two dimensions: positive coping styles and negative coping styles. This research takes 522 college students from ordinary universities in Jilin Province as the research objects, analyzes some characteristics of current college students' coping styles, and finds that college students are more likely to adopt negative coping styles when coping with stress.

Keywords: Coping Styles; General Situation; College Students

\section{Description of problem}

In 2012, The Commission on Youth surveyed teens aged 15 to 24 and found that more than 30\% of respondents felt stressed $^{[1]}$. Different people have their coping strategies and methods under different stress conditions. These coping strategies and methods constitute coping styles that reflects personal characteristics (Gibson \& Letinberg, 2001 ${ }^{[2]}$; Liang, 2006 ${ }^{[3]}$ ). Stress is an intangible thing in life, it helps to motivate people to maintain good performance, but stress can also cause physical and mental illness that harms the body and affects oneself (Wilks, 2008) ${ }^{[4]}$. Therefore, in the face of high stress, what kind of coping styles is adopted determines whether college students can survive the difficult situation and improve the academic effect.

Coping styles were the core content of research on coping with stress in the past 40 years. Coping styles was the interaction between individuals and stressful situations. To solve problems, individuals made cognitive and behavioral efforts. The coping styles are the individual's adjustment behavior to the changes in the real environment. The coping styles are the individual's adjustment behavior to the changes in the real environment. The main function is to adjust the role of stress events, including changing the assessment of stress events and adjusting the physical or emotional response related to events. Therefore, dividing coping styles into positive coping styles and negative coping styles is acceptable to most people (Parkes, 1990). ${ }^{[5]}$ Positive coping styles are conducive to problem solving, which in turn is good for physical and mental health, while negative coping styles can harm physical and mental health.

\section{General situation of coping styles of college students}

In order to analyze the general situation of coping styles of college students, this research adopted a simple coping styles questionnaire compiled by Xie, Yaning $(1998)^{[6]}$ to investigate 522 college students in ordinary universities in Jilin Province. There were two dimensions of coping styles: positive coping styles and negative coping styles. The results are as follows:

Copyright @ 2020 Zhihui Gong

doi: 10.18282/le.v9i7.1453

This is an open-access article distributed under the terms of the Creative Commons Attribution Non-Commercial License

(http://creativecommons.org/licenses/by-nc/4.0/), which permits unrestricted non-commercial use, distribution, and reproduction in any medium, provided the original work is properly cited. 
Table 1 General Situation of coping styles

\begin{tabular}{cccccc}
\hline & $\mathrm{N}$ & Min & Max & Mean & SD \\
1. Positive coping styles & 522 & 0.00 & 3.00 & 1.43 & .72 \\
2. Negative coping styles & 522 & 0.00 & 3.00 & 1.47 & .71 \\
\hline
\end{tabular}

It could be seen from Table 1 that there were 522 effective samples in this research. There were two dimensions of coping styles: positive coping styles and negative coping styles. From the research results, the average score of negative coping styles $(\mathrm{M}=1.47)$ was higher than the average score of positive coping styles $(\mathrm{M}=1.43)$.

When faced with academic stress, college students adopted more negative coping styles than positive coping styles, that is, college students tended to relieve their troubles by smoking, drinking, taking medicine, or even choosing to escape or imagine that miracles will occur to comfort themselves. This is inconsistent with the findings of Zhu, Yumei (2011) ${ }^{[7]}$ and Yao, Qi (2015). ${ }^{[8]}$ The reasons may be the first, the current Chinese college students are mostly onlychild, and the market economy has strengthened their autonomy and competition consciousness. They are eager to innovate, promote themselves, have rich emotions, and are often "self-centered". They often have poor interpersonal skills and do not get along well with their classmates and lack of guidance and support from mentors and friends, causing them to be psychologically lonely. Many students have poor self-control ability, when they encounter uncomfortable things, see unreasonable things, easily lose their temper, and make extreme behavior. Second, because college students are at a critical stage of development, both physical and psychological are not very mature, and they do not have enough ability to face all difficulties. So when they have various difficulties, they often take some irrational ways to deal with them. Third, with the improvement of the economic level and the improvement of family economic status, parents can give more help and support to their children. When a child encounters a problem, parents will help the child to solve the problem, leading to the college students' ability of the solution problem declines, and they don't know how to treat it properly in difficult situations, so they often adopt negative ways to face it.

\section{Conclusion}

The results of this research indicated that college students were more likely to adopt negative coping styles when they encountered stressful events or difficulties. Individual coping styles are influenced by both innate factors and acquired environment, so we must cultivate the attitude of college students to actively face stress events, and take effective measures, such as letting yourself release stress through some beneficial sports or cognition and maintaining good emotions will also improve college students' ability to deal with problems, ease stress, and thus improve academic achievement.

\section{References}

1. Commission on Youth Secretariat (2012). "Hong Kong youth development indicators study", accessed on February 3, 2016, available online at: http://www.coy.gov.hk/filemanager/template/common/images/archive/research/youth_ report_20111123_c.pdf.

2. Gibson, B. L., Litenberg, H. (2001). The impact of sexual abuse and stigma on methods of coping with sexsual assault among undergraduate women. Child Abuse \& Neglect, 25, 1343-1361.

3. Liang, B. Y. (2006). Mental Stress, Coping and Health: A Clinical Psychological Study of Stress and Coping. Beijing: Education Science Press, 11.

4. Wilks, S. E. (2008). Resilience amid academic stress: The moderating impact of social support among social work students. Advances in social work, 9(2), 106-125.

5. Parkes, K. R. (1990). Coping, negative affectivity, and the work environment: Additive and interactive predictors of mental health. Journal of Applied Psychology, 75(4), 399-409.

6. Xie, Yaning. (1998). Reliability and Validity of the Simple Coping Style Scale. Chinese Journal of Clinical Psychology, (02), 53-54.

7. Zhu,Yumei. (2011). Research on the relationship between stress perception, psychological resilience and coping style of college students (Master's thesis), Harbin Engineering University, China.

8. Yao, Qi. (2015). Research on the relationship among subjective well-being, coping styles and psychological resilience of college student volunteers (Master's thesis), Yangzhou University, China. 